\title{
Autopsias realizadas por el Dr. Salvador Allende en el Hospital Carlos van Buren de Valparaíso, Chile.
}

\author{
(Autopsies performed by Dr. Salvador Allende at Carlos van Buren \\ Hospital of Valparaíso, Chile.)
}

Salomón Pérez Gaete ${ }^{1}$, Valentina Rodríguez Badilla ${ }^{1}$, Myriam Castillo ${ }^{2}$, Rodrigo Cruz Choappa ${ }^{3 *}$.

${ }^{1}$ Alumnos de Medicina Universidad de Valparaíso. ${ }^{2}$ Biblioteca y Patrimonio Hospital Carlos van Buren de Valparaíso. ${ }^{3}$ Cátedra de Medicina Interna Universidad de Valparaíso, Servicio de Medicina Interna Hospital Carlos van Buren de Valparaíso.

*Autor para correspondencia: rcruzchoappa@gmail.com

Palabras clave: Allende, autopsias, Valparaíso

Keywords: Allende, autopsies, Valparaíso

\section{RESUMEN}

Introducción: entre los años 1933 y 1937, el Dr. Salvador Allende trabajó con regularidad en el servicio de Anatomía Patológica del Hospital Carlos van Buren de Valparaíso (HCVB), realizando autopsias en los casos de muertes de causa médica.

Materiales y método: se revisaron los libros del servicio de Anatomía Patológica del HCVB del año 1937 y se seleccionaron las autopsias realizadas por el Dr. Allende. Los libros y autopsias de los años 1933 a 1936 no pudieron ser encontrados. Los datos demográficos de los pacientes se traspasaron a una planilla Excel. Los resultados se expresaron en números y porcentajes.

Resultados: el Dr. Allende realizó 54 autopsias durante un periodo de 5 meses, lo que correspondió al 12,6\% de las realizadas ese año. De los 54 pacientes, $48(88,8 \%)$ correspondieron al sexo masculino, con un promedio de edad de 46 (9-97) años. La mayoría tenían nacionalidad chi- lena y estaban cesantes. E1 53,7\% de los casos falleció por una causa infecciosa y las más frecuentes fueron la neumonía y la tuberculosis. La concordancia entre los diagnósticos clínicos y de Anatomía Patológica fue de un 70,3\%.

Conclusiones: se encontró solo una parte de las autopsias realizadas por el Dr. Allende en el HCVB. La mayoría de los pacientes fallecidos fueron jóvenes del sexo masculino y cesantes. Las principales causas de muerte fueron la neumonía y la tuberculosis.

\section{ABSTRACT}

Introduction: Dr. Salvador Allende often worked between the years 1933 and 1937 for the pathology department in the Carlos van Buren Hospital, performing autopsies in the medical cause death cases.

Materials and Methods: the pathology department books from 1937 were reviewed sear- 
ching for the autopsies performed by Dr. Allende and those were selected. The books and autopsies of the years 1933-1936 could not be found. The demographic data about patients were transfered into an excel spreadsheet. The results were expressed as numbers and percentages.

Results: Dr. Allende performed 54 autopsies over a period of 5 months, which corresponded to $12.6 \%$ of all the autopsies performed that year. Of the 54 patients, 48 were male $(88.8 \%)$, with an average age of 46 (9-97) years. Most of them had Chilean nationality and unemployed. 53.7\% of studied cases died by an infectious cause being the most common pneumonia and tuberculosis. The concordance between clinical diagnoses and pathology department were $70.3 \%$.

Conclusions: Only a small part of the autopsies performed by Dr. Salvador Allende in HCVB was found. Most deceased patients were young men and unemployed. The main causes of death were pneumonia and tuberculosis.

\section{INTRODUCCIÓN}

Salvador Allende Gossens nació en Santiago el 26 de junio de 1908 (1). Su padre, Salvador Allende Castro, fue un destacado abogado y periodista que ocupó varios cargos en la administración pública. Su madre, Laura Gossens Uribe, hija de un inmigrante belga radicado en concepción (1). Su abuelo, Ramón Allende Padin ("el rojo"), fue un destacado médico y político radical que llegó a ser diputado y senador de Chile $(1,2)$.

Sus estudios básicos y secundarios los realizó en el Liceo de Tacna, Instituto Nacional, Liceo de Valdivia y el Liceo Eduardo de la Barra de Valparaíso (2). En 1926 ingresó a estudiar Medicina en la Universidad de Chile, titulándose en el año 1932 con su tesis " Higiene mental y delincuencia" $(1,2,3)$.
Participó en la creación de diversas organizaciones sociales y partidos políticos, fue ministro de sanidad, diputado y senador. En 1970 fue elegido presidente de la República de Chile apoyado por los partidos de la unidad popular, siendo derrocado por un golpe de estado cívicomilitar en el año 1973, luego del cual hubo una dictadura que duró 17 años $(1,2,4)$.

Entre los años 1933 y 1937, el Dr. Salvador Allende trabajó con cierta regularidad en el servicio de Anatomía Patológica del Hospital Carlos van Buren de Valparaíso (HCVB), realizando autopsias en los casos de muertes de causa médica (5). Este trabajo es muy poco conocido y sus protocolos de autopsias han permanecido en la biblioteca del HCVB solo como recuerdo de su paso, sin un análisis, sin mostrar la realidad de los muertos de los años mencionados.

Los objetivos del presente estudio fueron describir demográficamente a los pacientes fallecidos y autopsiados por el Dr. Salvador Allende en el año 1937, conocer sus causas de muerte y determinar la coincidencia entre los diagnósticos clínicos y de anatomía patológica.

\section{MATERIALES Y MÉTODOS}

Estudio descriptivo histórico. Se revisaron los libros del servicio de Anatomía Patológica del año 1937 y se seleccionaron los protocolos de autopsias realizadas por el Dr. Allende (imagen 1 y 2). Los libros y protocolos de autopsias de los años 1933 a 1936 no pudieron ser encontrados.

Recolección de datos: se confeccionó una planilla Excel que incluyó los antecedentes de los pacientes: sexo, edad, nacionalidad, profesión u oficio, fecha de ingreso al hospital, diagnóstico clínico y de anatomía patológica. 
Análisis: Los resultados se expresan en números y porcentajes, además se exponen en tablas y gráficos.

Confidencialidad: Se revisaron los libros de acuerdo con las recomendaciones de Biblioteca del HCVB para evitar daños del material históri- co. No se incluyeron en las planillas nombres ni otros datos que pudieran vulnerar la privacidad de los pacientes ya fallecidos o familiares.

El presente trabajo contó con la autorización del Comité Científico del HCVB.

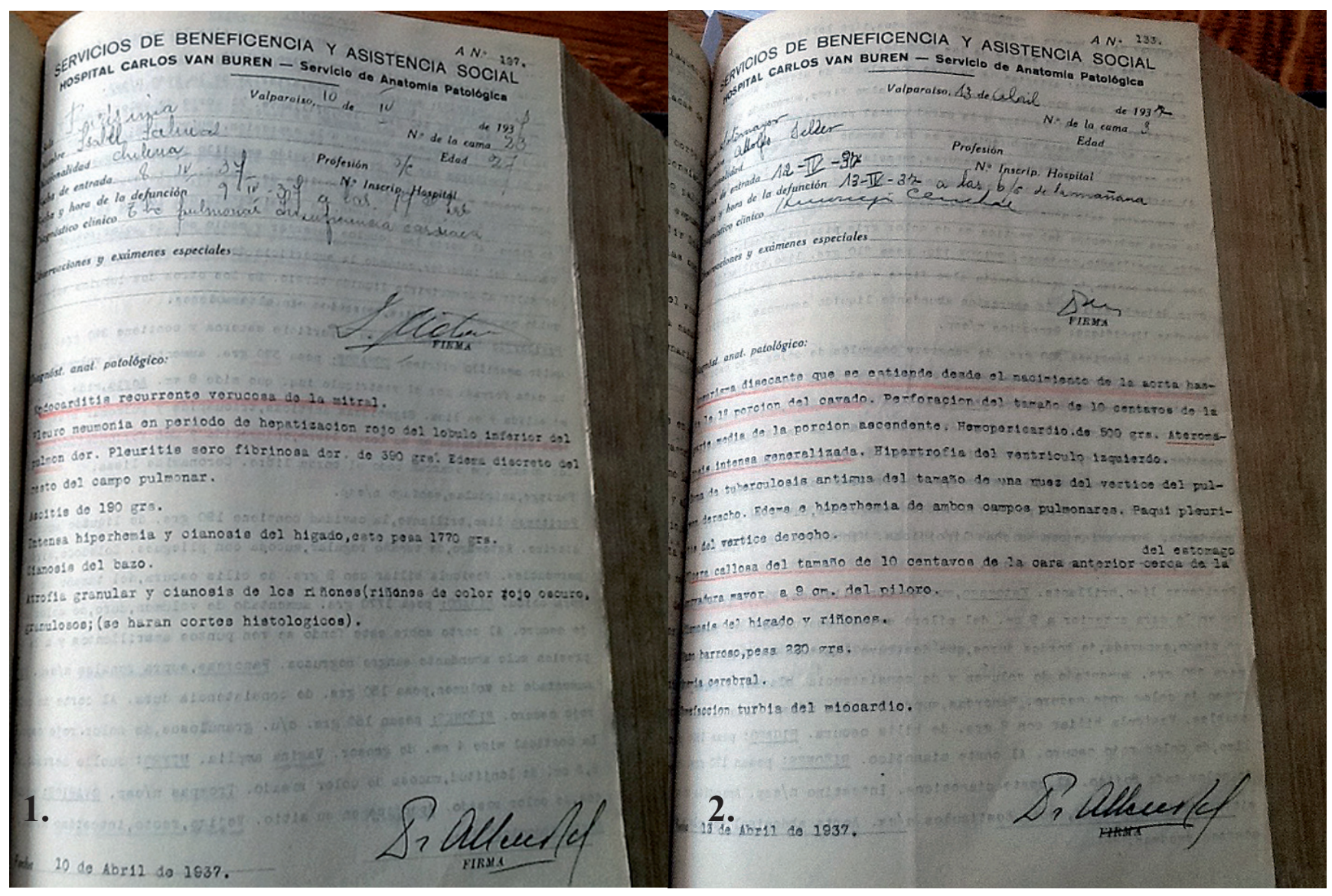

Figura 1. Se muestra protocolo de autopsia de un paciente con diagnóstico de endocarditis mitral y pleuroneumonía derecha.

Figura 2. Se muestra protocolo de autopsia de un paciente con diagnóstico de aneurisma disecante de la aorta.

\section{RESULTADOS}

Durante el año 1937 se realizaron 427 autopsias en total. De estas, el Dr. Allende realizó 54 durante un periodo de 5 meses (enero hasta mayo), lo que corresponde al 12,6\% del total. De estos, 48 correspondieron al sexo masculino $(88,8 \%)$, con un promedio de edad de 46 (9-97) años. Cincuenta $\mathrm{y}$ un pacientes eran de nacionalidad chilena, 1 alemán y en 2 casos no se consignó. Los oficios de los pacientes eran múltiples; carpinteros, obreros, comerciantes, empleados, agricultores, etc., sin embargo, muchos se encontraban cesantes (tabla 1). El $53,7 \%$ de los casos falleció por una causa 
infecciosa (tabla 2), siendo las más frecuentes la neumonía y la TBC en todas sus formas clínicas. Dentro de las causas no infecciosas destacó la causa cardiovascular y la neoplásica (tabla 3).

Tabla 1. Oficios de pacientes autopsiados.

\begin{tabular}{|l|l|}
\hline Oficios & $\mathbf{n}$ \\
\hline Cesante & 7 \\
Oarpintero & 5 \\
Comero & 4 \\
Empleado & 4 \\
Vendedor & 3 \\
Agricultor & 3 \\
Otros & 21 \\
No se consigna & 4 \\
Total & 54 \\
\hline
\end{tabular}

Tabla 2. Causas de muerte.

\begin{tabular}{|l|l|}
\hline Causas & $\mathbf{n}$ \\
\hline Infecciosa & 29 \\
No Infecciosa & 23 \\
Otros & 2 \\
Total & 54 \\
\hline
\end{tabular}

Tabla 3. Muertes de causa no infecciosa.

\begin{tabular}{|l|l|}
\hline Causas & $\mathbf{n}$ \\
\hline Cardiovascular & 11 \\
Neoplásica & 7 \\
Digestiva & 4 \\
Metabólica & 1 \\
Otros & 2 \\
Total & 25 \\
\hline
\end{tabular}

La coincidencia entre los diagnósticos clínicos y las autopsias fue de $70,3 \%$.

\section{DISCUSION}

Según Mario Amorós (5), el expresidente Allende encontró trabajo regular como asistente del servicio de Anatomía Patológica del HCVB desde mediados de 1933, además de trabajar en el policlínico denominado "el socorro socialista". El ex presidente declaró haber realizado en total aproximadamente 1500 autopsias (5), sin embargo, no se sabe con exactitud cuántas realizó. En este trabajo, solo encontramos evidencia de su trabajo en los registros de protocolos de autopsias del año 1937, esto probablemente debido a la pérdida de los registros de los años anteriores.

La mayoría de los pacientes autopsiados fueron hombres jóvenes, situación que coincide con los datos de mortalidad de los años 30 publicados por el Instituto Nacional de Estadísticas (INE), donde la población de hombres que llegaba a adulto mayor era menor que la de mujeres (6).

Los pacientes autopsiados tenían distintos oficios o estaban cesantes, lo que también coincide con lo publicado por el INE que muestra que en los años de nuestro estudio un $43 \%$ de los hom- 
bres y un $46 \%$ de las mujeres eran analfabetas y que los oficios informales eran los trabajos más frecuentes (6).

La principal causa de muerte fueron las infecciones y dentro de ellas la neumonía y la TBC en sus distintas formas clínicas. Esto estaba determinado probablemente por la pobreza, hacinamiento y marginalidad de la población, además de la falta de antimicrobianos, los que aparecieron a fines de los años 30 en el caso de las sulfas, a mediados de los años 40 la penicilina (7) y a fines de los 40 la estreptomicina (8).

Dentro de las causas no infecciosas, las de etiología cardiovascular fue la más frecuente, seguido de la neoplásica. Estas son las más frecuentes en la mortalidad tanto en hombres como en mujeres en el Chile actual, a diferencia de lo que ocurría en los años de este estudio (9).

Hubo una alta concordancia de diagnóstico clínico y anatomopatológico, lo que muestra la alta sospecha clínica de los médicos en un tiempo donde la tecnología médica era precaria (10).

Las autopsias realizadas por el ex presidente que se pudieron rescatar son una fracción de las que realmente realizó, sin embargo, reflejan la realidad cruda de esos años y que probablemente contribuyó a forjar su carácter y compromiso social que siempre lo caracterizó.

\section{REFERENCIAS}

1. Yocelevzky R. Biografía del querido hermano Salvador Allende Gossens. En: Salvador Allende Gossens en la memoria de sus hermanos masones. Santiago, Chile: Editorial Occidente; 2012. p. 18-26.

2. Vuskovic S. Algunos datos biográficos del presidente Allende. En: Allende en el mundo. Valparaiso, Chile: Editorial arte \& gráfica; 2002. p: 6-10.

3. Allende S. Higiene Mental y delincuencia. Tesis para optar al título de médico cirujano. Universidad de Chile 1933. Santiago, Chile: Ediciones Chile América CESOC; 2005. p: 1-159.

4. Cruz-Coke R. Síntesis biográfica del doctor Salvador Allende G. Rev Med Chile. 2003; 131(7): 809-14.

5. Amorós Mario. Médico, socialista y masón. Allende La biografía. Barcelona, España: Ediciones BSA; 2013. p:39-42.
6. INE. Hombres y mujeres de Chile. Enfoque estadístico. 2010; p: 1-8. Disponible en www.ine.cl [Consultado el 2 de mayo de 2016].

7. Ibarra C, Parada M. Producción de Penicilina en Chile entre 1944 y 1954. Rev Chil Infectol 2015; 32(1): 88-96.

8. Lederman W. La tuberculosis después del descubrimiento de Koch. Rev Chil Infectol 2003; 4850 .

9. INE. Evolución de la mortalidad en Chile según causas de muerte y edad.1990-2007. 2010.p: 2226. Disponible en www.ine.cl [Consultado el 11 de mayo de 2016].

10. Laborde M. Punto de quiebre a mediados de los años 30. En Medicina Chilena En El Siglo XX (Reseña Histórica). Santiago, Chile: Alvimpress Impresores. 2002:55-64. 